\title{
Proton decay testing low energy supersymmetry with precision gauge unification
}

\author{
Stefan Pokorski, ${ }^{1,2}$ Krzysztof Rolbiecki, ${ }^{1}$ and Kazuki Sakurai ${ }^{1}$ \\ ${ }^{1}$ Institute of Theoretical Physics, Faculty of Physics, University of Warsaw, \\ Ludwika Pasteura 5, PL-02-093 Warsaw, Poland \\ ${ }^{2}$ Theoretical Physics Department, CERN, CH-1211 Geneva 23, Switzerland
}

(Received 2 August 2017; published 28 February 2018)

\begin{abstract}
We show that gauge coupling unification in supersymmetry (SUSY) models can make a nontrivial interconnection between collider and proton decay experiments. Under the assumption of precise gauge coupling unification in the Minimal Supersymmeric Standard Model, with negligible grand unified theories threshold corrections, the low energy SUSY spectrum and the unification scale are intertwined, and the lower bound on the proton lifetime can be translated into upper bounds on SUSY masses. We find that the current limit on $\tau\left(P \rightarrow \pi^{0} e^{+}\right)$already excludes gluinos heavier than $\sim 70$ (120 and 200) $\mathrm{TeV}$ if their mass ratio to winos, $R \equiv M_{3} / M_{2}$, is $\sim 1$ ( 3 and 7), respectively. Next generation nucleon decay experiments are expected to bring these upper bounds down to $\sim 5$ (10 and 15) $\mathrm{TeV}$ for $R \sim 1$ ( 3 and 7).
\end{abstract}

DOI: 10.1103/PhysRevD.97.035027

Proton decay would be the key evidence for grand unified theories (GUTs) [1]. Among possible decay channels, a special role is played by the $p \rightarrow \pi^{0} e^{+}$mode for which the dominant contribution may come from the $D=6$ operators depending almost exclusively on the $X$, $Y$ boson mass and the unified gauge coupling. Unification of the gauge couplings in the minimal supersymmeric Standard Model (MSSM) as a link between the supersymmetric spectrum and the GUT spectrum and a window to the GUT physics has been studied intensively. Most such works have focused on constraining the GUT spectrum making simple assumptions on the low energy supersymmetry (SUSY) spectrum. In this study, we take the opposite approach, very similar to the one proposed in the early Ref. [2] with similar motivation.

Following several studies [3,4], we assume that the unification of the gauge couplings is precise within the MSSM without threshold corrections of GUT scale particles. In fact, such a situation can be realized in a class of extra dimensional GUT models, proposed as a solution to the doublet-triplet splitting problem. In such a scenario, the relevant superheavy particles are in general mass degenerate around the compactification scale, leading to small threshold corrections. In particular, in a 6-D orbifold GUT model that breaks the GUT symmetry nonlocal by Wilson

Published by the American Physical Society under the terms of the Creative Commons Attribution 4.0 International license. Further distribution of this work must maintain attribution to the author(s) and the published article's title, journal citation, and DOI. Funded by SCOAP ${ }^{3}$. lines, the threshold correction exactly vanishes when the size of two extra dimensions are the same $[5,6]{ }^{1}$

On the other hand, the GUT threshold corrections in conventional models depend on the mass ratios between superheavy particles forming incomplete multiplets (such as the colored Higgs and the $X, Y$ bosons in the minimal $\mathrm{SU}(5)$ ). If those superheavy particles have hierarchical mass spectra, the threshold corrections can become large enough to spoil "the success of gauge coupling unification in the MSSM" [5,8]. We stress again that all conclusions derived in this paper are subject to the assumption of the precision gauge coupling unification within the MSSM.

Under the assumption of precise gauge coupling unification (GCU), we show that the low energy SUSY spectrum and the unification scale are intertwined, and the lower bound on the proton lifetime $\tau_{p \rightarrow \pi^{0} e^{+}}$can be translated into upper bounds on SUSY masses. ${ }^{2}$ This leads to an interesting interconnection between the proton decay experiments and the collider searches, particularly in view of the future progress on both fronts, in cornering supersymmetric spectrum from above and from below.

In general, the $p \rightarrow K^{+} \nu$ mode, induced by the $D=5$ operators generated by the colored Higgs exchange

\footnotetext{
${ }^{1}$ Small but not necessarily vanishing threshold corrections are expected in extra dimensional orbifold GUT models with boundary condition GUT breaking [7].

${ }^{2}$ Unlike other upper bounds on SUSY masses based on the arguments of the Higgs boson mass [9] or the neutralino relic abundance [10], these bounds depend neither on the ratio of the Higgs vacuum expectation values, $\tan \beta \equiv v_{u} / v_{d}$, nor the assumption of $R$-parity conservation and the thermal history of the universe.
} 
diagrams, also gives a strong constraint on the colored Higgs mass and low energy SUSY spectrum as well as the structure of the Higgs sector in the GUT models [11]. However, this mode is highly model-dependent, and several mechanisms have been known to suppress it independently of the GCU [12]. ${ }^{3}$ We therefore do not consider this mode in this paper for simplicity. In any case, the constraints derived from the $p \rightarrow \pi^{0} e^{+}$mode can be applied independently from the $D=5$ proton decay constraints.

We use the two-loop renormalization group equations (RGEs) for the running of the gauge couplings, which we solve numerically. The solution can be written as [13]

$$
\frac{2 \pi}{\alpha_{i}(Q)}=\frac{2 \pi}{\alpha_{i}\left(m_{Z}\right)}-b_{i} \ln \left(\frac{Q}{m_{Z}}\right)+s_{i}+\gamma_{i}+\Delta_{i},
$$

where $\alpha_{1} \equiv \frac{5}{3} \alpha_{Y}, i=1,2,3$ represents the gauge group, $b_{i}=\left(\frac{33}{5}, 1,-3\right)$ are the one-loop $\beta$-function coefficients for the MSSM and

$$
s_{i} \equiv \sum_{\eta} b_{i}^{\eta} \ln \left(\frac{m_{\eta}}{m_{Z}}\right)
$$

represent the threshold corrections of low energy SUSY particles. The variables $m_{\eta}$ and $b_{i}^{\eta}$ denote the mass and the contribution to $b_{i}$ from the superpartner $\eta$. The $\gamma_{i} \equiv-\frac{1}{2} \sum_{j} \frac{b_{i j}}{b_{j}} \ln \left(\frac{\alpha_{j}(Q)}{\alpha_{j}\left(m_{Z}\right)}\right)$ accounts for the two-loop contribution with $b_{i j}$ being the two-loop $\beta$-function coefficients. ${ }^{4}$ The $\Delta_{i}$ represents the effect of the top Yukawa coupling and the conversion factor between $\overline{\mathrm{MS}}$ and $\overline{\mathrm{DR}}$ schemes. ${ }^{5}$

In the special case where all SUSY particles are mass degenerate at $M_{s}$, the threshold correction can be written as $s_{i}=\delta_{i} \ln \left(M_{S} / m_{Z}\right)$ with $\delta_{i} \equiv\left(b_{i}-b_{i}^{\mathrm{SM}}\right)$, where $b_{i}^{\mathrm{SM}}=$ $\left(\frac{41}{10},-\frac{19}{6},-7\right)$ are the one-loop $\beta$-function coefficients for the Standard Model (SM). In this case, precision gauge unification $\alpha_{1}(Q)=\alpha_{2}(Q)=\alpha_{3}(Q) \equiv \alpha_{G}^{*}$ is achieved by the particular values, $M_{s}=M_{s}^{*}, Q=M_{G}^{\mathrm{deg} *}$, satisfying

$$
\frac{2 \pi}{\alpha_{G}^{*}}=\frac{2 \pi}{\alpha_{i}\left(m_{Z}\right)}-b_{i} \ln \left(\frac{M_{G}^{\mathrm{deg} *}}{m_{Z}}\right)+\delta_{i} \ln \left(\frac{M_{s}^{*}}{m_{Z}}\right)+\gamma_{i}+\Delta_{i}
$$

for all $i$. It should be borne in mind that the quantities $M_{s}^{*}$, $M_{G}^{\mathrm{deg} *}$ and $\alpha_{G}^{*}$ are not variables but constants defined as the solution to the above three simultaneous equations.

Coming back to the general case, let us decompose the vector $s_{i}$ into three independent vectors as [4]

\footnotetext{
${ }^{3}$ The $D=5$ proton decays are suppressed also in the class of models discussed in [6,7].

${ }^{4}$ At the unification scale $\left(M_{G}\right)$, it can be approximated as $\gamma_{i}=-\frac{1}{2} \sum_{j} \frac{b_{i j}}{b_{j}} \ln \left(1+\frac{b_{j} \alpha_{G}}{2 \pi} \ln \frac{M_{G}}{m_{Z}}\right)$. One can solve $\gamma_{i}$ iteratively by updating $M_{G}$ and $\alpha_{G}$.

${ }^{5}$ For the treatment of $\Delta_{i}$, see for example [14].
}

$$
s_{i}=\delta_{i} \ln \left(\frac{T}{m_{Z}}\right)+b_{i} \ln \Omega+C
$$

The solution to this set of equations is given by

$$
\begin{aligned}
\ln \left(\frac{T}{m_{Z}}\right) & =\frac{v_{i} s_{i}}{D} \\
\ln \Omega & =\frac{u_{i} s_{i}}{D} \\
C & =\frac{\epsilon_{i j k} \delta_{j} b_{i} s_{k}}{D}
\end{aligned}
$$

where summation is understood for the repeated indices and $\epsilon_{i j k}$ is the antisymmetric tensor and

$$
\begin{gathered}
v=\left(\begin{array}{c}
b_{2}-b_{3} \\
-b_{1}+b_{3} \\
b_{1}-b_{2}
\end{array}\right), \quad u=\left(\begin{array}{c}
-\delta_{2}+\delta_{3} \\
\delta_{1}-\delta_{3} \\
-\delta_{1}+\delta_{2}
\end{array}\right), \\
D=b_{2} \delta_{1}-b_{3} \delta_{1}-b_{1} \delta_{2}+b_{3} \delta_{2}+b_{1} \delta_{3}-b_{2} \delta_{3} .
\end{gathered}
$$

Plugging the concrete values of $b_{i}, \delta_{i}$ and $b_{i}^{\eta}$ into these expressions, one gets

$$
\begin{aligned}
T= & {\left[M_{3}^{-28} M_{2}^{32}\left(\mu^{4} m_{A}\right)^{3} X_{T}\right]^{\frac{1}{19}}, } \\
\Omega= & {\left[M_{3}^{-100} M_{2}^{60}\left(\mu^{4} m_{A}\right)^{8} X_{\Omega}\right]^{\frac{1}{288}}, } \\
C= & \frac{125}{19} \ln M_{3}-\frac{113}{19} \ln M_{2}-\frac{40}{19} \ln \mu-\frac{10}{19} \ln m_{A} \\
& +\sum_{i=1 \ldots 3}\left[\frac{79}{114} \ln m_{\tilde{d}_{R i}}-\frac{10}{19} \ln m_{\tilde{l}_{i}}-\frac{121}{114} \ln m_{\tilde{q}_{i}}\right. \\
& \left.+\frac{257}{228} \ln m_{\tilde{u}_{R i}}+\frac{33}{76} \ln m_{\tilde{e}_{R i}}\right],
\end{aligned}
$$

with

$$
\begin{gathered}
X_{T} \equiv \prod_{i=1 \ldots 3}\left(\frac{m_{\tilde{l}_{i}}^{3}}{m_{\tilde{d}_{R i}}^{3}}\right)\left(\frac{m_{\tilde{q}_{i}}^{7}}{m_{\tilde{e}_{R i}}^{2} m_{\tilde{u}_{R i}}^{5}}\right), \\
X_{\Omega} \equiv \prod_{i=1 \ldots 3}\left(\frac{m_{\tilde{l}_{i}}^{8}}{m_{\tilde{d}_{R i}}^{8}}\right)\left(\frac{m_{\tilde{q}_{i}}^{6} m_{\tilde{e}_{R i}}}{m_{\tilde{u}_{R i}}^{7}}\right) .
\end{gathered}
$$

We ignore the phases of SUSY breaking parameters since they do not contribute to the running of gauge couplings. In most models, the sfermion contributions to $T$ and $\Omega$ are negligible (i.e. $X_{T} \sim X_{\Omega} \sim 1$ ). In particular, these contributions vanish if the masses are degenerate within the $\mathrm{SU}(5)$ multiplets, $\overline{\mathbf{5}}_{i}=\left(\tilde{d}_{R}^{c}, \tilde{l}\right)_{i}, \mathbf{1 0}_{i}=\left(\tilde{q}, \tilde{u}_{R}^{c}, \tilde{e}_{R}^{c}\right)_{i}$. One can explicitly check that for a degenerate spectrum, $\ln \Omega=C=0$. 
To see roles of $T, \Omega$ and $C$ in GCU, we substitute Eq. (4) into Eq. (1) and obtain

$\frac{2 \pi}{\alpha_{i}(Q)}=\frac{2 \pi}{\alpha_{G}^{*}}-b_{i} \ln \left(\frac{Q}{\Omega M_{G}^{\mathrm{deg} *}}\right)+\delta_{i} \ln \left(\frac{T}{M_{s}^{*}}\right)+C$,

where Eq. (3) has also been used. ${ }^{6}$ It is clear that the exact unification for the general case is obtained when the rhs becomes $i$-independent, that is at $T=M_{s}^{*}$ [15] and the exact unification scale is given by

$$
M_{G}=\Omega M_{G}^{\mathrm{deg} *} .
$$

The unified gauge coupling is related to that of the degenerate case as

$$
\alpha_{G}^{-1}=\alpha_{G}^{*-1}+\frac{C}{2 \pi} .
$$

Away from the exact unification, we define a candidate unification scale $M_{U}$ and a semiunified coupling $\alpha_{U}$ by

$$
\alpha_{1}\left(M_{U}\right)=\alpha_{2}\left(M_{U}\right) \equiv \alpha_{U}
$$

This scale can be computed from the low energy spectrum as

$$
M_{U}=\Omega M_{G}^{\operatorname{deg} *}\left(T / M_{s}^{*}\right)^{\frac{\delta_{1}-\delta_{2}}{b_{1}-b_{2}}},
$$

where $\frac{\delta_{1}-\delta_{2}}{b_{1}-b_{2}}=-\frac{25}{84} \simeq-0.3$, and at this scale the gauge couplings are given by

$$
\frac{2 \pi}{\alpha_{i}\left(M_{U}\right)}=\frac{2 \pi}{\alpha_{G}^{*}}+\left(\delta_{i}-b_{i} \frac{\delta_{1}-\delta_{2}}{b_{1}-b_{2}}\right) \ln \left(\frac{T}{M_{s}^{*}}\right)+C .
$$

Using this formula, a measure of gauge coupling unification, which we define as $\epsilon_{3} \equiv\left(\alpha_{3}\left(M_{U}\right)-\alpha_{U}\right) / \alpha_{U}$, is calculated as

$$
\epsilon_{3}=\frac{\alpha_{G}^{*}}{2 \pi} Y \ln \left(\frac{T}{M_{s}^{*}}\right)+\cdots,
$$

where the dots represent higher order terms of $\frac{\alpha_{G}^{*}}{2 \pi}$ and

$Y \equiv \frac{b_{1}\left(\delta_{2}-\delta_{3}\right)+b_{2}\left(-\delta_{1}+\delta_{3}\right)+b_{3}\left(\delta_{1}-\delta_{2}\right)}{b_{1}-b_{2}}=\frac{19}{14}$.

It is interesting that $\epsilon_{3}$ depends only on $T$ at the leading order [15].

\footnotetext{
${ }^{6}$ Strictly speaking, $\gamma_{i}$ and $\Delta_{i}$ do not cancel out in this expression, since the unification scales and the unified couplings are different between the general and the degenerate SUSY cases. However, the differences are of higher order and can be neglected.
}

All numerical scans presented in this paper use a two-loop RGE code including the effect of the top Yukawa coupling, following [14]. We use $\tan \beta=10$, but a variation of $\tan \beta$ results in negligible effects. The SUSY breaking parameters are uniformly scanned in the logarithmic scale within $\left[m_{\min }\right.$, $\left.10^{3} \mathrm{TeV}\right]$. We take $m_{\min }=1.5 \mathrm{TeV}$ for $M_{3}$ and $200 \mathrm{GeV}$ for $M_{2}, \mu$ and $m_{A}$. The sfermion masses are assumed to be universal $\left(\equiv m_{\tilde{f}}\right)$ for simplicity and $m_{\min }=1 \mathrm{TeV}$ is used. We also vary $\alpha_{3}\left(m_{Z}\right)=0.1184(7)$, according to the $1-\sigma$ uncertainty. At the central value of $\alpha_{3}\left(m_{Z}\right)$, numerically, $M_{s}^{*}=2.08 \mathrm{TeV}, M_{G}^{\mathrm{deg} *}=1.27 \times 10^{16} \mathrm{GeV}$ and $\alpha_{G}^{*-1}=25.5$.

We show in Fig. 1 the result of our numerical scan. The top plot in Fig. 1 confirms the predicted relation Eq. (20) (dashed line). Hereafter, we keep the points that have precise GCU, $\left|\epsilon_{3}\right|<0.1 \%$, and discard them otherwise. We see that the precision GCU occurs only when the SUSY masses are arranged such that $T$ computed by Eq. (9) is within a certain range, $[1,4] \mathrm{TeV}$, centred around $2 \mathrm{TeV}$. The width of $T$ for precision GCU is generated by two factors: a variation of $M_{s}^{*}$ corresponding to the uncertainty on $\alpha_{3}\left(m_{Z}\right)$ and a small violation of $T=M_{s}^{*}$ caused by up to $0.1 \%$ deviation from the exact unification, $\epsilon_{3}=0$. The middle plot shows the correlation between $\Omega$ and the exact unification scale, $M_{G}$. The color of points represents a typical SUSY scale $\sqrt{M_{3} M_{2}}$. One can see that heavy SUSY tends to have a small unification scale. For the PeV scale SUSY with $\sqrt{M_{3} M_{2}} \sim 10^{3} \mathrm{TeV}, M_{G}$ is reduced by a factor of 5 compared to the TeV scale one. The bottom plot confirms the predicted relation Eq. (16) (dashed line). The color-code indicates a SUSY scale, $\left(M_{3} M_{2} m_{\tilde{f}}\right)^{\frac{1}{3}}$. We see that high scale SUSY tends to predict a smaller unified coupling, $\alpha_{G}$, but the variation is small and only up to $\sim 10 \%$ between the $\mathrm{TeV}$ and PeV scale SUSY mass points.

An interesting observation follows from the last two plots of Fig. 1. High scale SUSY, where the unification scale is lower, in general leads to a rapid proton decay, $p \rightarrow e^{+} \pi^{0}$. This is because the rate $\Gamma\left(p \rightarrow e^{+} \pi^{0}\right)$ scales as $\alpha_{G} /\left(M_{G}\right)^{4}$, where the $X, Y$ boson mass is identified as the unification scale, since the precise gauge unification implies all GUT particles forming incomplete GUT multiplets (e.g. $X, Y$ bosons and colored Higgs) have the same mass, $M_{G}$. Turning this around, the lower limit on $M_{G}$ from the proton lifetime measurement (if found, bearing in mind that the variation of $\alpha_{U}$ is small) can place upper bounds on the masses of SUSY particles. Let us denote this lower limit by $M_{P D}: M_{G}>M_{P D}$. Then, eliminating $M_{3}$ from Eq. (10) by using Eqs. (9) and (18) gives us

$$
M_{2}^{\frac{4}{5}}\left(\mu^{4} m_{A}\right)^{\frac{1}{25}}<M_{S}^{*}\left(\frac{M_{P D}}{M_{G}^{\operatorname{deg} *}}\right)^{-\frac{2016}{475}} X_{\mathrm{EW}}^{\frac{1}{25}},
$$

with

$$
X_{\mathrm{EW}} \equiv \prod_{i=1 \ldots 3}\left(\frac{m_{\tilde{l}_{i}}}{m_{\tilde{d}_{R i}}}\right)\left(\frac{m_{\tilde{q}_{i}}^{7}}{m_{\tilde{e}_{R i}}^{3} m_{\tilde{u}_{R i}}^{4}}\right)
$$



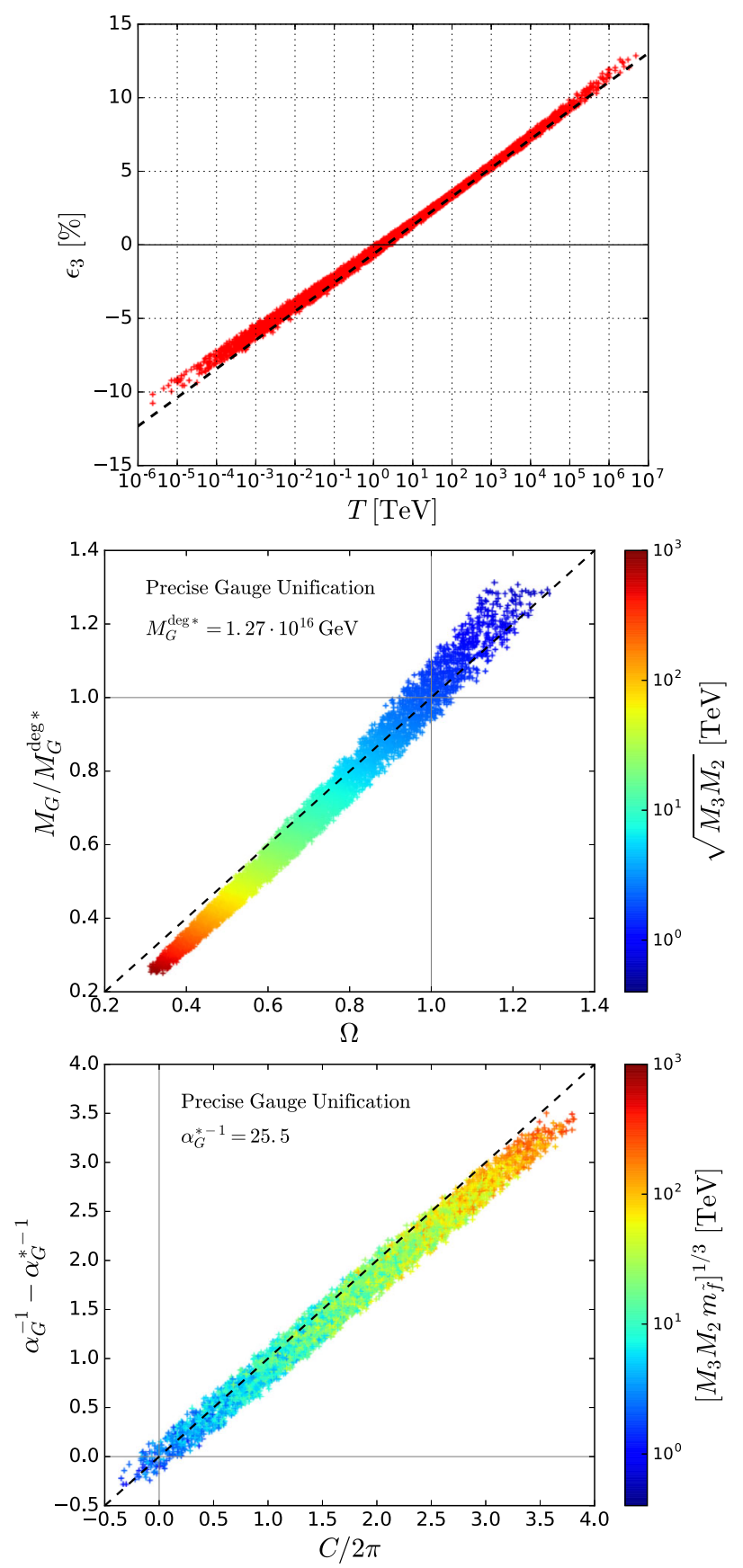

FIG. 1. Scan of SUSY particle masses projected onto the $\left(T, \epsilon_{3}\right)$ (top), the $\left(\Omega, M_{G} / M_{G}^{\mathrm{deg} *}\right)$ (middle) and $\left(C / 2 \pi, \alpha_{G}^{-1}-\alpha_{G}^{*-1}\right)$ (bottom) planes. In the middle and bottom plots, the precise gauge unification $\left(\left|\epsilon_{3}\right|<0.1 \%\right)$ is required (corresponding to the blue band in the top plot), and the color-codes represent typical SUSY scales $\sqrt{M_{3} M_{2}}$ and $\left(M_{3} M_{2} m_{\tilde{f}}\right)^{\frac{1}{3}}$, respectively. The dashed lines represent the two-loop relations Eqs. (20), (15) and (16) for the top, middle and bottom plots, respectively.

where $M_{s}^{*}=2.08 \mathrm{TeV}$ and $M_{G}^{\mathrm{deg} *}=1.27 \times 10^{16} \mathrm{GeV}$ are the constants. This implies that the smallest mass in the lhs is bounded from above by the rhs of Eq. (22). When this bound is saturated, $M_{2}=\mu=m_{A}$. The upper

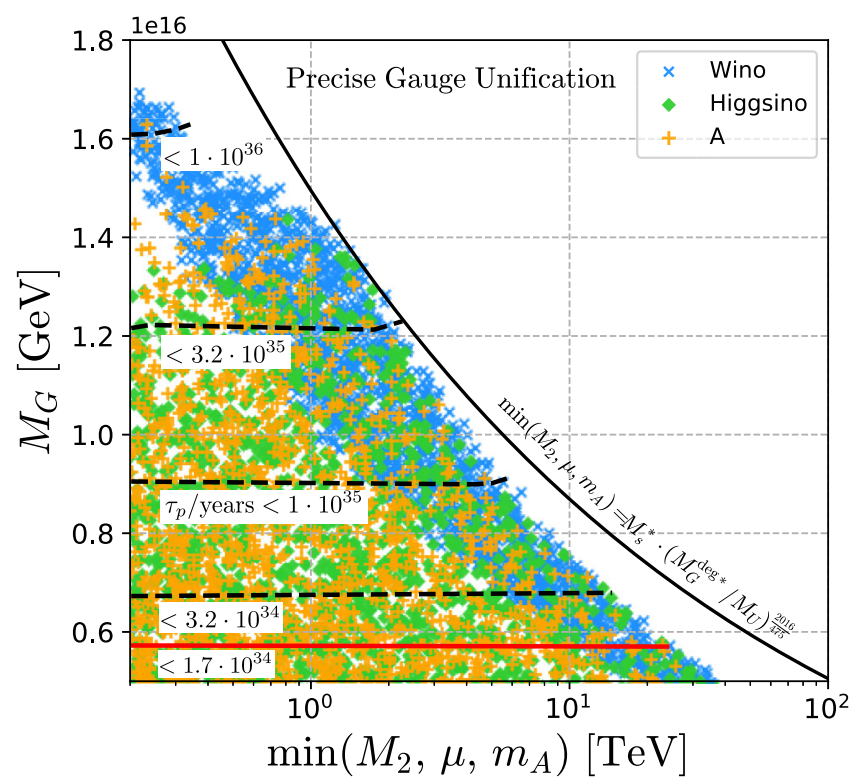

FIG. 2. Points with precise gauge unification $\left(\left|\epsilon_{3}\right|<0.1 \%\right)$ projected onto the $\min \left(M_{2}, \mu, m_{A}\right)$ vs $M_{G}$ plane. The blue, green and orange points correspond to the points where $M_{2}, \mu$ and $m_{A}$ is the smallest among them, respectively. The regions below a black-dashed or a red-solid line are excluded by the quoted future or current limits on the proton lifetime. The black-solid line corresponds to the upper bound Eq. (22) for $T=M_{S}^{*}$ and $M_{P D}=M_{G}$.

limit on the individual parameters are obtained, for example, as

$$
M_{2}<M_{s}^{*}\left(\frac{\mu^{4} m_{A}}{M_{s}^{* 5}}\right)^{-\frac{1}{20}}\left(\frac{M_{P D}}{M_{G}^{\operatorname{deg} *}}\right)^{-\frac{504}{95}} X_{\mathrm{EW}}^{\frac{1}{20}}
$$

In this expression the rhs is bounded from above by the experimental lower limit on $\mu$ and $m_{A}$.

The upper bound Eq. (22) is observed in our numerical scan shown in Fig. 2, where the smallest of $M_{2}, \mu$ and $m_{A}$ is plotted in the $x$-axis. The blue, green and orange points correspond to the cases where $M_{2}, \mu$ and $m_{A}$ is the lightest among the three, respectively. A tendency is observed that $M_{2}$ is close to the upper limit if $M_{2}$ is the lightest. This is due to the higher power for $M_{2}$ in Eq. (22) than for $\mu$ and $m_{A}$. At each point we calculate $\tau_{p \rightarrow \pi^{0} e^{+}}$based on $[16,17]^{7}$ using $\alpha_{G}$ and $M_{G}$ obtained by the two-loop RGE code. The horizontal black-dashed and red-solid lines represent the boundaries where all points below them have the lifetime shorter than the quoted values. In particular, the region below the red line is excluded by the current limit: $\tau_{p \rightarrow \pi^{0} e^{+}}>1.7 \times 10^{34}$ years [19].

${ }^{7}$ The calculation of $\tau_{p \rightarrow \pi^{0} e^{+}}$is not completely model independent. For example, $\tau_{p \rightarrow \pi^{0} e^{+}}$in flipped SU(5) models is smaller by $1 /\left[1+\left(1+V_{u d}\right)^{2}\right] \sim 1 / 5$ than in conventional models [18]. 
The upper bound on the gluino mass can be found by eliminating $\mu^{4} m_{A}$ in Eq. (10) by using Eq. (9) as

$$
M_{3}<M_{s}^{*}\left(\frac{M_{2}}{M_{s}^{*}}\right)^{-1}\left(\frac{M_{P D}}{M_{G}^{\operatorname{deg} *}}\right)^{-\frac{216}{19}} X_{\tilde{g}}^{\frac{1}{4}}
$$

with

$$
X_{\tilde{g}} \equiv \prod_{i=1 \ldots 3}\left(\frac{m_{\tilde{u}_{R i}} m_{\tilde{e}_{R i}}}{m_{\tilde{q}_{i}}^{2}}\right)
$$

As previously, the rhs of Eq. (25) is bounded from above by the experimental lower limit on the wino mass.

If the SUSY breaking mechanism is specified, the ratio of gluino and wino masses is usually predicted. Assuming the value of $R \equiv M_{3} / M_{2}$, the following upper bounds can be derived:

$$
\begin{gathered}
M_{3}<M_{S}^{*} R^{\frac{1}{2}}\left(\frac{M_{P D}}{M_{G}^{\mathrm{deg} *}}\right)^{-\frac{108}{19}} X_{\tilde{g}}^{\frac{1}{8}}, \\
M_{2}<M_{s}^{*} R^{-\frac{1}{2}}\left(\frac{M_{P D}}{M_{G}^{\operatorname{deg} *}}\right)^{-\frac{108}{19}} X_{\tilde{g}}^{\frac{1}{8}}, \\
\left(\mu^{4} m_{A}\right)^{\frac{1}{5}}<M_{s}^{*} R^{2}\left(\frac{M_{P D}}{M_{G}^{\mathrm{deg} *}}\right)^{\frac{144}{90}} \cdot X_{\mu}^{\frac{1}{10}},
\end{gathered}
$$

where

$$
X_{\mu} \equiv \prod_{i=1 \ldots 3}\left(\frac{m_{\tilde{l}_{i}}^{2}}{m_{\tilde{d}_{R i}}^{2}}\right)\left(\frac{m_{\tilde{q}_{i}}^{4}}{m_{\tilde{e}_{R i}} m_{\tilde{u}_{R i}}^{3}}\right) .
$$

We show in Fig. 3 our scan in the $\left(M_{3}, M_{2}\right)$ plane with the color-code indicating $\left(\mu^{4} m_{A}\right)^{\frac{1}{5}}$. As previously, the black-dashed and red-solid lines represent the future and current bounds on $\tau_{p \rightarrow \pi^{0} e^{+}}$. It is evident that $M_{3}$ and $M_{2}$ are highly sensitive to the proton lifetime and constrained by it from above. This is in direct contrast to collider searches, constraining these parameters from below. Unlike $M_{3}$ and $M_{2}, \mu$ and $m_{A}$ are almost insensitive to the proton lifetime, which follows from the lower power of $M_{P D}$ in Eq. (29). On the other hand, they are highly sensitive to $R$. In particular, $\mu$ is typically a TeV for $R \sim 1$ whereas it is $\mathcal{O}(100) \mathrm{TeV}$ for $R \sim 7$. The implication of this to naturalness and phenomenology are studied in detail in $[3,4]$.

It is remarkable that the current proton lifetime limit already excludes the gluino and wino masses larger than 70 and $70 \mathrm{TeV}$ for $R \sim 1$ (compressed SUSY), 120 and $40 \mathrm{TeV}$ for $R \sim 3$ (e.g. CMSSM, GMSB) and 200 and $30 \mathrm{TeV}$ for $R \sim 7$ (e.g. AMSB), respectively. Next generation nucleon decay experiments are expected to improve the current

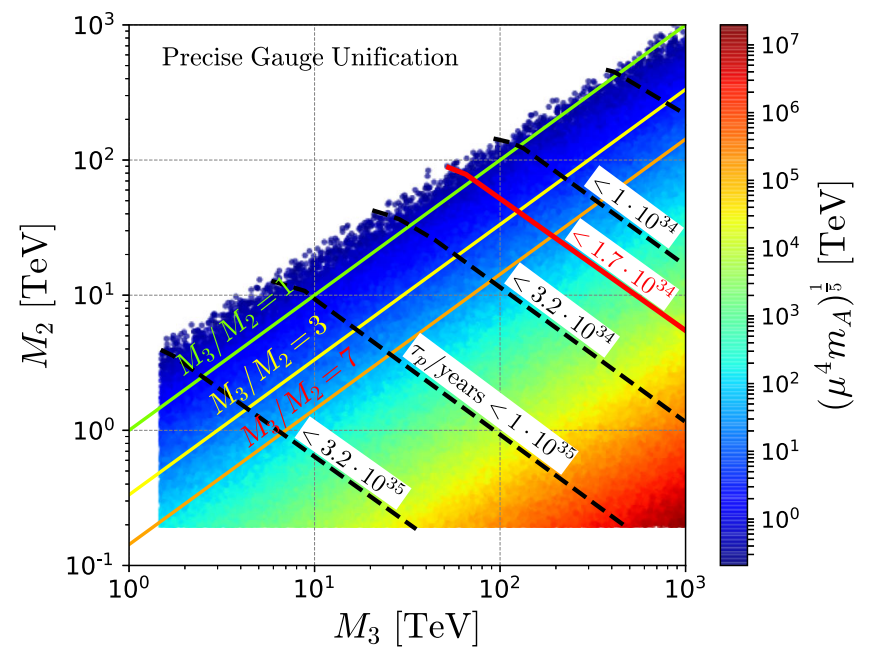

FIG. 3. Points with precise gauge unification $\left(\left|\epsilon_{3}\right|<1 \%\right)$ projected onto the $\left(M_{3}, M_{2}\right)$ plane. The color-code shows $\left(\mu^{4} m_{A}\right)^{\frac{1}{5}}$. The regions above the black-dashed and red-solid lines are excluded by the quoted future or current limits on $\tau_{p \rightarrow \pi^{0} e^{+}}$. The three diagonal lines correspond to $R \equiv M_{3} / M_{2}=1,3$ and 7 from top to bottom. In this plot the upper boundaries of $\mu$ and $m_{A}$ scans are extended up to $5 \times 10^{7} \mathrm{TeV}$.

$\tau_{p \rightarrow \pi^{0} e^{+}}$limit by a factor of ten [16], which will result in tightening the upper bounds on gluino and wino masses further down to $\left(M_{3}, M_{2}\right) \lesssim(5,5) \mathrm{TeV}$ for $R \sim 1$, $(10,3) \mathrm{TeV}$ for $R \sim 3$ and $\left(M_{3}, M_{2}\right) \lesssim(15,2) \mathrm{TeV}$ for $R \sim 7$. These bounds are close to the lower mass limits $\left(M_{3}, M_{2}\right) \gtrsim(10,2.7) \mathrm{TeV}[20,21]$, which are expected to be obtained at future $100 \mathrm{TeV}$ hadron-hadron colliders.

We have checked how these upper bounds are weakened if we relax the condition on the precision GUT to $\left|\epsilon_{3}\right|<1 \%$. In such a case, we found the current upper bounds on the gluino and wino masses to be 90 and $90 \mathrm{TeV}$ for $R \sim 1,180$ and $60 \mathrm{TeV}$ for $R \sim 3$ and 280 and $40 \mathrm{TeV}$ for $R \sim 7$, respectively.

It is worth noting that the above conclusion is robust against the details of the rest of the SUSY spectrum as long as sfermion masses are not split significantly within the SU(5) multiplets. In fact, the impact of nondegenerate sfermion masses on the superpartner mass bounds can be understood by, for instance, Eqs. (26) and (27). Typically, the squark and slepton masses split due to the RGEs and become $m_{\tilde{u}_{R_{i}}} \sim m_{\tilde{q}_{i}} \sim 3 m_{\tilde{e}_{R_{i}}}$ at the TeV scale. In this case, the gluino mass bound is even tightened by a factor of 0.7 for a fixed $R$. Our conclusion also holds even if new particles, which are singlets under the SM gauge group, are added to the MSSM, as in the case of the next-to-minimal supersymmetric Standard Model.

We have investigated the link between the proton lifetime $\tau_{p \rightarrow \pi^{0} e^{+}}$and the supersymmetric spectrum under the assumption of small GUT thresholds. It has been shown that most of the allowed mass range of gluinos and winos will be probed by future collider and proton lifetime 
experiments. It is straightforward to extend this analysis to concrete models with sizeable threshold corrections. In this case, the result will depend on the details of the GUT models (see e.g. [22]).

\section{ACKNOWLEDGMENTS}

We thank Zackaria Chacko, Kiwoon Choi, Emilian Dudas, Sebastian Ellis, Shigeki Matsumoto, Stuart Raby and James Wells for helpful discussion. We thank Stuart Raby for a discussion on the GUT threshold corrections. The work of S. P. and K. S. is partially supported by the National Science Centre, Poland, under research Grants No. DEC-2014/15/B/ST2/02157 and No. DEC-2015/18/ $\mathrm{M} / \mathrm{ST} 2 / 00054$. The work of K. R. and K. S. is supported by the National Science Centre (Poland) under Grant No. 2015/19/D/ST2/03136.
[1] For early references see, P. Langacker, Phys. Rep. 72, 185 (1981).

[2] N. V. Krasnikov, JETP Lett. 61, 245 (1995); Phys. Lett. B 392, 365 (1997); arXiv:hep-ph/9710520.

[3] S. Raby, M. Ratz, and K. Schmidt-Hoberg, Phys. Lett. B 687, 342 (2010).

[4] S. Krippendorf, H. P. Nilles, M. Ratz, and M. W. Winkler, Phys. Rev. D 88, 035022 (2013); H. P. Nilles, Adv. High Energy Phys. 2015, 1 (2015).

[5] S. Raby, Supersymmetric Grand Unified Theories (Springer International Publishing, Gewerbestrasse, 2017).

[6] A. Hebecker, J. High Energy Phys. 01 (2004) 047; A. Hebecker and M. Trapletti, Nucl. Phys. B713, 173 (2005); A. Anandakrishnan and S. Raby, Nucl. Phys. B868, 627 (2013).

[7] K. R. Dienes, E. Dudas, and T. Gherghetta, Nucl. Phys. B537, 47 (1999); Phys. Lett. B 436, 55 (1998); Y. Kawamura, Prog. Theor. Phys. 105, 999 (2001); L. J. Hall and Y. Nomura, Phys. Rev. D 64, 055003 (2001); A. Hebecker and M. Trapletti, Nucl. Phys. B713, 173 (2005).

[8] S. A. R. Ellis and J. D. Wells, Phys. Rev. D 91, 075016 (2015); 96, 055024 (2017).

[9] G. F. Giudice and A. Strumia, Nucl. Phys. B858, 63 (2012); M. Ibe and T. T. Yanagida, Phys. Lett. B 709, 374 (2012); E. Bagnaschi, G. F. Giudice, P. Slavich, and A. Strumia, J. High Energy Phys. 09 (2014) 092.

[10] N. Arkani-Hamed, A. Delgado, and G. F. Giudice, Nucl. Phys. B741, 108 (2006); J. Hisano, S. Matsumoto, M. Nagai, O. Saito, and M. Senami, Phys. Lett. B 646, 34 (2007); J. Ellis, F. Luo, and K. A. Olive, J. High Energy Phys. 09 (2015) 127.
[11] J. Hisano, H. Murayama, and T. Yanagida, Nucl. Phys. B402, 46 (1993); Phys. Rev. Lett. 69, 1014 (1992).

[12] H. Georgi, Phys. Lett. 108B, 283 (1982); B. Grinstein, Nucl. Phys. B206, 387 (1982); A. Masiero, D. V. Nanopoulos, K. Tamvakis, and T. Yanagida, Phys. Lett. 115B, 380 (1982); K. S. Babu, I. Gogoladze, and Z. Tavartkiladze, Phys. Lett. B 650, 49 (2007).

[13] L. J. Hall, Nucl. Phys. B178, 75 (1981); M. B. Einhorn and D. R. T. Jones, Nucl. Phys. B196, 475 (1982); U. Amaldi, W. de Boer, and H. Furstenau, Phys. Lett. B 260, 447 (1991).

[14] P. Langacker and N. Polonsky, Phys. Rev. D 47, 4028 (1993).

[15] M. Carena, S. Pokorski, and C. E. M. Wagner, Nucl. Phys. B406, 59 (1993).

[16] K. S. Babu et al., arXiv:1311.5285.

[17] B. Bajc, J. Hisano, T. Kuwahara, and Y. Omura, Nucl. Phys. B910, 1 (2016).

[18] H. Murayama and A. Pierce, Phys. Rev. D 65, 055009 (2002).

[19] V. Takhistov (Super-Kamiokande Collaboration), arXiv: 1605.03235.

[20] T. Cohen, T. Golling, M. Hance, A. Henrichs, K. Howe, J. Loyal, S. Padhi, and J. G. Wacker, J. High Energy Phys. 04 (2014) 117.

[21] M. Low and L. T. Wang, J. High Energy Phys. 08 (2014) 161.

[22] J. Hisano, T. Kuwahara, and N. Nagata, Phys. Lett. B 723, 324 (2013); A. Hebecker and J. Unwin, J. High Energy Phys. 09 (2014) 125. 